\title{
Hacia la inclusión de los pequeños agricultores, población vulnerable, en programas de vigilancia toxicológica mediante la implementación de marcadores biológicos de fácil acceso en zonas rurales de Colombia
}

\author{
Karen Rocio López Castro, Universidad Militar Nueva Granada, Colombia \\ Maira Fernanda Zambrano Carrillo, Universidad Militar Nueva Granada, Colombia
}

\begin{abstract}
Resumen: En la actualidad la información referente a la morbilidad causada por intoxicación crónica a causa de actividades asociadas al trabajo con agroquímicos en la población colombiana es muy pobre, adicionalmente no existen evidencias de programas de seguimiento y control del nivel de exposición a tóxicos como medida de prevención en la aparición de enfermedades crónicas de origen toxicológico en trabajadores del sector agrícola informal. Los pequeños agricultores son una población extremadamente vulnerable que ha sido pasada por alto en los programas nacionales de vigilancia y control de intoxicaciones ocasionadas por plaguicidas. Esta investigación, pretende demostrar la pertinencia del uso de biomarcadores de fácil acceso en pequeños agricultores dentro de programas de prevención y control de intoxicaciones crónicas generadas por plaguicidas como aporte en la humanización del trabajo.
\end{abstract}

Palabras clave: plaguicidas, ocupacional, toxicidad, agricultura, biomarcadores

\begin{abstract}
Currently, the information regarding morbidity caused by chronic poisoning from activities associated with agrochemicals exposure in Colombian population is poor; further, there is no evidence of programs neither to monitor or control the level of exposure to pesticides nor to prevent the onset of chronic disease in agricultural workers of the informal sector. Small farmers are an extremely vulnerable population that has been overlooked in national surveillance pesticide poisoning programs. This research aims to demonstrate the relevance of using easily accessible biomarkers to evaluate small farmers as part of prevention and control of chronic pesticide poisoning and thus contribute to work humanization.
\end{abstract}

Keywords: Pesticides, Occupational, Toxicity, Agriculture, Biomarkers

\section{Introducción}

$\mathrm{L}$

a producción agrícola en Colombia representa el $8 \%$ de la producción interna bruta junto con la ganadería, silvicultura y la caza, distribuidos en todos los territorios del país. En el desarrollo de esta actividad económica se vinculan un número de trabajadores con vinculación formal e informal que se someten a ciertos riesgos ocupacionales dentro de los cuales se encuentran los riesgos químicos, que pueden generar el desarrollo de enfermedades profesionales causadas por la exposición a sustancias químicas y sus derivados, descritas en la tabla de enfermedades profesionales para Colombia y adoptada por el Ministerio de Protección Social mediante el decreto 2566 del 7 de julio de 2009.

En el desarrollo de esta actividad económica se vinculan un número de trabajadores con vinculación formal e informal que se someten a ciertos riesgos ocupacionales dentro de los cuales se encuentran los riesgos químicos, que pueden generar el desarrollo de enfermedades profesionales causadas por sustancias químicas y sus derivados, descritas en la tabla de enfermedades profesionales para Colombia y adoptada por el Ministerio de Protección Social mediante el decreto 2566 del 7 de julio de 2009. Dentro de las principales fuentes de riesgo químico se encuentran los plaguicidas cuyos principios activos se constituyen como tóxicos con elevada incidencia en la morbilidad y 
mortalidad de intoxicaciones agudas y crónicas, que afectan principalmente a los trabajadores del sector agrícola como los esencialmente expuestos a los efectos nocivos de dichos compuestos ya que son utilizados para matar, reducir y repeler varios tipos de plagas que afectan el desarrollo de los cultivos (OMS, 2004).

Dentro de las principales fuentes de riesgo químico se encuentran los plaguicidas inhibidores de la colinesterasa, cuyos principios activos se constituyen como tóxicos con elevada incidencia en la morbilidad y mortalidad de intoxicaciones agudas y crónicas, que afectan principalmente a los trabajadores del sector agrícola como los esencialmente expuestos a los efectos nocivos de dichos compuestos ya que son utilizados para matar, reducir y repeler varios tipos de plagas que afectan el desarrollo de los cultivos (OMS, 2004).

En España el Instituto Nacional de Higiene y Seguridad en el Trabajo, en sus normas NTP 660 y 661 de 1999 propone el Control Biológico de Trabajadores Expuestos a Plaguicidas, el cual se basa en la determinación de la colinesterasa plasmática, eritrocitaria, GPT y GGT. Estos dos últimos parámetros bioquímicos son dos enzimas relacionados con los efectos en el tejido hepático, de carácter totalmente inespecífico, que se alteran por la exposición importante a cualquier agente hepatotóxico. Siendo este país pionero en el control y vigilancia de la intoxicación crónica por exposición a plaguicidas.

En el año 2005 Chaves J. y colaboradores publican su estudio de exposiciones laborales a agentes carcinogénicos y plaguicidas en Costa Rica, siendo la primera vez que el CAREX (Carcinogen Exposure) europeo es aplicado en otros países, en el cuál se abordan los plaguicidas como factores carcinogénicos, pero sin tomar en cuenta el impacto tóxico de los mismos de acuerdo a lo anteriormente propuesto en España.

En el 2006, la Comisión Europea propone "La Política Comunitaria para Uso Sostenible de los Plaguicidas Origen de la Estrategia", la cual enfatiza en los daños que pueden generar los plaguicidas en la salud y el medio ambiente, sin embargo no se plantean planes de seguimiento y control biológico en los trabajadores.

En Colombia las intoxicaciones agudas por plaguicidas se constituyen como un evento de salud pública del que hasta el año 2002 no se tenían datos reportados y consolidados: "En la actualidad no se encuentran fuentes primarias que puedan dar cuenta del comportamiento de este grupo de eventos (intoxicaciones/envenenamientos) en años recientes y específicos para Intoxicaciones Agudas por Plaguicidas -IAP-. La morbilidad en todos los años no se ha elaborado de la misma manera, incluso en los últimos años no se encuentra reportada" (INS, 2011).

Desde el año 2002 se han generado programas de capacitación liderados por el Ministerio de Protección social y el Instituto Nacional de Salud, enfocados a la prevención de intoxicaciones agudas y manejo de las mismas en los centros hospitalarios, sin embargo no toda la población agrícola en Colombia tiene acceso a dichos programas, lo cual permite el aumento no sólo de intoxicaciones agudas, sino que da paso a la aparición de enfermedades profesionales de origen toxicológico que en la gran mayoría de los casos son diagnosticadas de forma tardía (OMS, INS, INSO, 2006).

En el 2006 Colombia en conjunto con Bolivia generaron el Proyecto de cooperación técnica entre Países para el fortalecimiento de la vigilancia en salud pública de los plaguicidas, donde repetidamente se le dio mayor relevancia al manejo de las intoxicaciones agudas, relegando el seguimiento a las intoxicaciones crónicas por dichos compuestos químicos (OMS, INS, INSO, 2006).

En 2010, el Instituto Nacional de Salud lanza el programa VEO de Vigilancia Epidemiológica de Plaguicidas Organofosforados y Carbamatos, cuyo principal objetivo consiste en determinar los niveles de actividad de acetilcolinesterasa en sangre de individuos con riesgo de exposición a plaguicidas organofosforados (OF) y carbamatos (C) e impulsar el desarrollo de acciones de promoción, prevención, seguimiento y control que disminuyan el efecto de estos tóxicos sobre la salud, sin embargo, en este programa no se incluye el apoyo de otros biomarcadores diferentes a la acetilcolinesterasa como apoyo diagnóstico del estado real de salud generalizada de los agricultores como herramientas para valorar el deterioro crónico de la salud de los trabajadores cuyas actividades se relacionan con el uso de plaguicidas (INS, 2011). 
En el 2011 el Instituto Nacional de Salud propone un nuevo protocolo de vigilancia y control de intoxicaciones por plaguicidas cuyo objetivo general es "realizar el seguimiento continuo y sistemático de los casos de intoxicaciones agudas por plaguicidas de acuerdo con los procesos establecidos para la notificación, recolección y análisis de los datos que permitan generar información oportuna, válida y confiable para orientar medidas de prevención y control del evento" (OMS, 2011), documento en donde se evidencia reiteradamente la priorización ante la intoxicación aguda y se deja de lado el efecto y seguimiento de intoxicaciones crónicas.

En la actualidad la información existente acerca de la toxicidad crónica a causa de actividades asociadas al trabajo en la población colombiana es muy pobre, adicionalmente no se evidencia la existencia de programas de seguimiento y control del nivel de exposición a tóxicos como medida de prevención en la aparición de enfermedades crónicas de origen toxicológico en los trabajadores del sector agrícola y mucho menos en los trabajadores informales del este sector.

No se encontraron evidencias de estudios del empleo de la medición de biomarcadores toxicológicos adicionales a la acetilcolinesterasa, como herramienta de control y vigilancia epidemiológica en intoxicaciones crónicas generadas por plaguicidas dentro de la población agrícola colombiana en ninguna de las bases de datos consultadas en el Ministerio de la Protección Social, OMS Secretarías Distritales y Departamentales de Salud, que permita obtener datos de diagnóstico temprano y prevención de enfermedades profesionales generadas por exposición a compuestos químicos específicamente plaguicidas; que podrían ser utilizados como herramientas de fortalecimiento en los programas de control y vigilancia en salud ocupacional, aportando a la humanización del trabajo agrícola en Colombia.

En Colombia, existen guías de manejo de individuos con signos de intoxicación aguda por exposición a plaguicidas organosfosforados, que incluye la realización de pruebas diagnósticas complejas como la colinesterasa eritrocitaria, realizadas en laboratorios de referencia a nivel urbano nacional, sin embargo no se proponen herramientas diagnósticas de fácil acceso para agricultores informales con recursos económicos limitados y pertenecientes al sector rural, cuyo acceso a servicios de salud corresponde a entidades hospitalarias de primer y segundo nivel de atención incapaces de realizar pruebas como la colinestrasa eritrocitaria. Esta investigación, pretende demostrar la pertinencia de la determinación del nivel de biomarcadores co-ayudadores como apoyo diagnóstico del estado de salud integral, en individuos con exposición crónica a organofosforados, de fácil acceso para poblaciones vulnerables cuyas actividades laborales se desarrollan en el área rural de Natagaima en el departamento de Tolima. Los resultados obtenidos muestran que existe necesidad de realizar seguimiento, vigilancia y control de enfermedades crónicas por exposición a organofosforados a través de herramientas científicas de fácil acceso para la población agrícola, con posible inclusión en las políticas públicas del país.

El objetivo de esta investigación era determinar la pertinencia y viabilidad del uso de otros biomarcadores diferentes a la acetilcolinesterasa eritrocitaria en una población agricultora del sector informal.

\section{Metodología}

\section{1.-Muestreo poblacional}

La selección de las poblaciones fue intencional o por conveniencia después de establecer contacto con la alcaldía y los agricultores del municipio de Natagaima. Las bases de datos de los trabajadores del sector agrícola en los municipios fueron solicitados a la Alcaldía Municipal, de las cuales se estimó la población expuesta. 


\section{2.-Encuesta}

Se aplicó una encuesta para indagar acerca del estado y antecedentes de salud, historia laboral y prácticas de higiene y seguridad durante la aplicación de plaguicidas en la población. La encuesta se diseñó teniendo en cuenta la GTC 45 de 2010, el decreto 1843 de 1991 y la encuesta CDC-NIOSH 2005.

\section{3.-Análisis de resultados}

Se analizaron los resultados en términos de porcentajes de las respuestas a cada pregunta de la encuesta aplicada a los participantes.

\section{4.-Consideraciones éticas}

El presente estudio corresponde a investigación con riesgo mínimo para los participantes según lo establecido por la resolución 008430 de 1993 del Ministerio de Salud de Colombia. Se protegió la privacidad de los participantes y los datos utilizando un sistema de codificación.

\section{Resultados}

La población agricultora informal encuestada corresponde a un grupo de 48 hombres entre los 21 y 78 años cuya principal actividad es el cultivo de arroz en el municipio de Natagaima- Tolima en Colombia.

Ninguno de los agricultores vinculados al estudio, posee certificación para ejecutar actividades asociadas al manejo de plaguicidas, además de presentar un elevado nivel de desconocimiento en el manejo y uso adecuado de los mismos.

El 77\% de la población encuestada presenta signos y síntomas como visión borrosa, cefalea y mareo, los cuales no han sido incluidos en ningún programa de vigilancia preventiva. Solamente el $11 \%$ utiliza todos los elementos de protección personal, siendo lo más común en el $28 \%$ de la población el uso de botas de caucho, gorro, pañuelo como protección respiratoria y una bolsa para cubrir la espalda. Los elementos de protección personal más usados son el gorro (94\%) y las botas de caucho (94\%) y los menos usados son los guantes (22\%) y el overol (17\%). Como justificación a no usar todos los elementos de protección individual, el $44 \%$ piensa que es por incomodidad, el $28 \%$ por factores económicos, el $17 \%$ desconocen la importancia de estos elementos y el $6 \%$ creen que no tienen una utilidad aparente.

En cuanto a medidas de higiene durante y después de las aplicaciones de plaguicidas el $6 \%$ reportó consumir alimentos durante la fumigación, el $22 \%$ consume bebidas, el $89 \%$ lava su ropa de trabajo en casa y el $89 \%$ toma una ducha al finalizar la aplicación.

Respecto al historial de incapacidades laborales, el 44\% manifestó haber suspendido labores por malestar durante la aplicación, el $28 \%$ haber acudido al médico al menos una vez por esta razón, al $84 \%$ nunca se les ha realizado algún examen de sangre como parte de programas preventivos de intoxicación con plaguicidas y al $100 \%$ de la población nunca se les ha realizado un análisis de colinesterasa eritrocitaria.

Los síntomas más comunes reportados por la población expuesta fueron en orden descendente visión borrosa (77\%), lagrimeo, sudoración profusa y dolor muscular (61\%), irritación ocular $(55 \%)$, pérdida de la memoria (50\%), rigidez muscular (44\%), dolor de cabeza y picazón en la piel (38\%). El consumo de alcohol y cigarrillo se reportó en el 11\% de la población.

\section{Análisis de resultados}

Se pudo evidenciar la gran deficiencia en el uso de elementos de protección individual en los aplicadores de plaguicidas quienes frecuentemente utilizan únicamente sombrero y botas de caucho, el uso de los demás elementos de protección personal como guantes, gafas, respirador y overol es 
reducido. Como principal justificación de los agricultores a este comportamiento está la incomodidad física o incomodidad térmica al usar estos elementos y además los altos costos de los mismos tal y como se ha reportado en otros estudios (Feola y Binder, 2010, MacFarlane et al., 2013) sin embargo, se sabe que hay una alta influencia de las normas sociales en las comunidades de agricultores que estarían influyendo en la deficiencia de uso de elementos de protección personal (Feola and Binder, 2010) como por ejemplo la percepción generalizada en la comunidad de que los elementos de protección personal son inútiles (Gomes et al., 1999). Los pequeños agricultores de las zonas rurales de los países en vía de desarrollo como Colombia, usualmente utilizan equipo de protección personal insuficiente o inadecuado y además tienden a utilizar plaguicidas innecesariamente o en exceso (Feola y Binder, 2010, Feola y Binder, 2010).

La normatividad colombiana establece que toda persona que aplique plaguicidas debe estar registrada y haber recibido capacitación teórico-práctica por parte de un ente educativo autorizado (Decreto 1843, 1991), sin embargo, ninguno de los agricultores encuestados recibió dicha capacitación ni tienen la certificación como "Aplicador de Plaguicidas". Dado que la mayoría de los cultivadores de arroz del Tolima son pequeños agricultores arrendatarios de las tierras que cultivan (Fedearroz, 2008), es poco frecuente que reciban capacitaciones o que estén incluidos en programas de salud ocupacional.

A pesar de manifestar su conciencia del riesgo, la mayoría de los agricultores no tienen conocimiento sobre prácticas de salud ocupacional como uso de elementos de protección personal, no comer o beber mientras hacen las aplicaciones o no llevar la ropa de trabajo a casa, y no realizan la disposición adecuada de los recipientes contaminados incrementando así el riesgo de exposición a plaguicidas. Este comportamiento está documentado en otros estudios en donde se evidencia que los agricultores tienen algunos conocimientos del riesgo de los plaguicidas pero un tienen un gran desconocimiento de medidas específicas para prevenir exposiciones ocupacionales (Barraza et al., 2011).

Los síntomas más reportados por los agricultores como visión borrosa, pérdida de la memoria y dolor de cabeza son inespecíficos, sin embargo pueden estar relacionados con exposición aguda o crónica a plaguicidas.

La población cultivadora de arroz estudiada pertenece a la zona arrocera centro de Colombia, caracterizada por una alta proporción de individuos indígenas mayores de 40 años los cuales son una población altamente vulnerable a desarrollar toxicidad por exposición aguda o crónica a plaguicidas durante sus labores agrícolas ya que nunca han estado inmersos en programas preventivos de salud ocupacional ni buenas prácticas agrícolas. Adicionalmente nunca se les ha realizado el examen de medición de colinesterasa eritrocitaria debido a que esta prueba no está disponible en el hospital municipal y además su remisión a un laboratorio especializado generaría altos costos que no son asumidos ni por el agricultor ni por el sistema de salud.

Colombia al igual que otros países latinoamericanos subdesarrollados muestra deficiencias en almacenamiento, desecho, uso inadecuado de elementos de protección personal y uso de plaguicidas restringidos, todo esto dentro de un contexto social y económico que hace muy difícil la implementación de programas de vigilancia y control del uso seguro de plaguicidas.

Dado que la población estudiada nunca ha sido objeto de programas de biomonitoreo, se hace necesaria la modificación de las estrategias nacionales de prevención de intoxicación por exposición ocupacional a plaguicidas para que incluyan a los pequeños agricultores en programas integrales de salud pública que contemplen la capacitación en aplicación segura de plaguicidas y especialmente en programas de salud ocupacional que no solo contemplen la medición de colinesterasa eritrocitaria sino también la medición de biomarcadores séricos de fácil acceso a nivel rural como las transaminasas hepáticas y marcadores de daño renal y cardíaco que han probado ser biomarcadores de efecto muy eficientes para la detección de estados subclínicos de patologías ocasionadas por la exposición de plaguicidas (Hernández, 2006). 


\section{Conclusiones}

La población de agricultores estudiada muestra una gran deficiencia en el manejo de prácticas adecuadas de salud ocupacional y buenas prácticas agrícolas lo cual hace que sea altamente vulnerable a sufrir los efectos de intoxicaciones agudas y crónicas por exposición a plaguicidas. Dadas las dificultades geográficas y económicas de las poblaciones de agricultores informales rurales en Colombia, en este trabajo se sugiere la pertinencia y viabilidad de la medición de otros biomarcadores diferentes a la acetilcolinesterasa los cuales pueden ser utilizados como biomarcadores de diagnóstico precoz de efectos bioquímicos causados por plaguicidas aún antes de que ocurran efectos adversos clínicos visibles y los cuales a diferencia de la colinesterasa eritrocitaria si están disponibles en los hospitales municipales.

Es necesario replicar el estudio en otras poblaciones y sectores productivos del país, para dar continuidad a la correlación clínica frente a hábitos y niveles de capacitación de los agricultores con el fin de generar impacto en las políticas públicas.

\section{Agradecimientos}

Este trabajo fue financiado por la Universidad Militar Nueva Granada mediante el proyecto (EES-1230). 


\section{REFERENCIAS}

Barraza, D., Jansen, K., Wendel, B. y Wesseling, C. (2011). Pesticide use in banana and plantain production and risk perception among local actors in Talamanca, Costa Rica. Environmental Research, 111, 708-717.

Chaves, J., Partanen, T., Wessling, C., Chaverri, F., Monge, P., Ruepert, C., Aragón, A., Kogevinas, M., Hogstedt, C. y Kaupinnen, T. (2005). TICAREX: Exposiciones laborales a agentes cancerígenos y plaguicidas en Costa Rica. Costa Rica: Archivos de Prevención de Riesgos Laborales, 8(1), 30-37.

Federación Nacional de Arroceros - Fondo Nacional del Arroz. (2008). Tercer censo nacional arrocero, Zona Centro. Colombia: Produmedios.

Feola, G. y Binder, C. (2010). Identifying and investigating pesticide application types to promote a more sustainable pesticide use. The case of smallholders in Boyacá, Colombia. Crop Protection, 29, 612-622.

Feola, G. y Binder, C. (2010). Why don't pesticide applicators protect themselves ? Exploring the use of personal protective equipment among Colombian smallholders. International Journal of Occupational and Environmental Health, 16(1), 11-23.

Guía Técnica Colombiana GTC 45. (2010). Guía Para la Identificación de los Peligros y la Valoración de los Riesgos en Seguridad y Salud Ocupacional. Colombia: Icontec Internacional, Consejo Colombiano de Seguridad.

Hernández, A., Gómez, A., Pérez, V., García-Lario, J., Peña, G., Gil, F., López, O., Rodrigo, L., Pinno, G. y Pla, A.(2006). Influence of exposure to pesticides on serum components and enzyme activities of citotoxicity among intensive agricultural farmers. Enviromental Research, 102(1), 70-76.

Instituto Nacional de Salud - INS. (2011). Programa de vigilancia epidemiológica de plaguicidas organofosforados y carbamatos VEO. Colombia: Grupo de Vigilancia y control de riesgo de factor ambiental.

Instituto Nacional de Salud - INS. (2011). Protocolo de vigilancia y control de intoxicaciones por plaguicidas. Colombia: Grupo de Vigilancia y control de riesgo de factor ambiental.

Comisión Europea. (2006). La política comunitaria para un uso sostenible de los plaguicidas Origen de la Estrategia. Luxemburgo: Comunidades Europeas.

McFarlane, E., Carey, R., Keegel, T., El-Zaemay, S. y Fritschi, L. (2013). Dermal exposure associated with occupational end use of pesticides and the role of protective measures. Safety and Health at Work, 4, 136-141.

Ministerio de Salud. Decreto 1843 de 1991. Por la cual se reglamentan parcialmente los títulos III, V, VI, VII Y XI de la ley 09 de 1979, sobre uso y manejo de plaguicidas. República de Colombia.

NTP 660. Control Biológico de Trabajadores Expuestos a plaguicidas (I): Aspectos Generales. Ministerio de Trabajo y Asuntos Sociales de España-Instituto Nacional de Seguridad e Higiene en el trabajo.

NTP 661. Control Biológico de Trabajadores Expuestos a plaguicidas (II): Técnicas Específicas. Ministerio de Trabajo y Asuntos Sociales de España-Instituto Nacional de Seguridad e Higiene en el trabajo.

OMS, INS, INSO. Colombia - Bolivia. (2006). Vigilancia en Salud Pública de los Plaguicidas. La Paz, Bolivia: C\&C Editores.

Organización Mundial de la Salud. (2011). Prevención de riesgos para la salud derivados del uso de plaguicidas en la agricultura. Serie Protección de la Salud de los Trabajadores № 1 . Busto Garolfo, Italia: OMS.

Organización Mundial de la Salud. (2004). Guidelines for Drinkingwater Quality, Vol. 1. Recommendations. Ginebra, Suiza: OMS. 
Occupational Health of Hired Farmworkers in the United States. (1999). National Agricultural Workers Survey Occupational Health Supplement. Department of Health and Human Service. Centers for Disease Control and Prevention National Institute for Occupational Safety and Health. CDC. NIOSH.

\section{SOBRE LAS AUTORAS}

Karen Rocio López Castro: Bióloga-Bacterióloga Magister en Ciencias. Docente e Investigadora. Programa Administración de la Seguridad y Salud Ocupacional. Universidad Militar Nueva Granada. Bogotá. Colombia.

Maira Fernanda Zambrano Carrillo: Bacterióloga y Laboratorista Clínico Especialista en Gerencia de Calidad. Docente e Investigadora Principal. Programa Administración de la Seguridad y Salud Ocupacional. Universidad Militar Nueva Granada. Bogotá. Colombia. 